\title{
Characterization of Multiple Refractive Scintillation Events in the Directions of Two Millisecond Pulsars
}

\section{Cognard}

\author{
LPCE-CNRS - 3A, Av Recherche Sc. - 45071 Orleans - France
}

J.-F. Lestrade

ARPEGES - Observatoire de Meudon - 92195 Meudon - France

\section{Introduction}

High-precision timing of the millisecond pulsars PSR B1937+21 and PSR B182124 are conducted at 1.4 and $1.7 \mathrm{GHz}$ with the high rate of observations of $10-15$ times per month at Nançay. Time Of Arrival (TOA) and pulsar flux density are both measured. The rms of the daily post-fit TOA residuals are at the level of $0.5 \mu \mathrm{s}$ and $3.0 \mu \mathrm{s}$, respectively. A characterization of refractive scintillation events produced by the Ionized Interstellar Medium (IISM) both in the TOA residuals and flux density variations is discussed.

\section{Observations and Analysis}

Timing at Nançay is conducted with a pre-detection dedispersion system. The analysis of the TOAs was done with our software AnTiOPE solving for the classical pulsar parameters. The flux densities of the pulsars are averaged over $\sim 30$ or $\sim 70$ minutes. Calibration of the system temperature and efficiency of the telescope is good at the $10 \%$ level and diffrative scintillation limits this calibration to a comparable level $(\sim 35$ scintels for PSR B1937+21 over 30 minutes and $7.5 \mathrm{MHz})$.

\section{Characterization of IISM refractive scintillation events}

IISM refractive scintillation events have been detected in recent years in the direction of extragalactic radio sources (Fiedler et al., 1987) via flux density variations and in direction of the millisecond pulsar PSR B1937+21, via both flux density variations and TOA variations (Cognard et al., 1993). When daily measurements are not carried out, the detection of a new refractive event is not as clear as in October 1989. We have experimented with a new method where a running rms of the post-fit TOA residuals is computed and plotted for comparison with the flux density variations. These plots should help in identifying refractive scintillation events. We have used several criteria other than the running rms but this latter one was the most satisfactory. The period over which the running rms is computed is $+/-20$ days around each TOA measurement after testing lower durations for which the lack of smoothing were producing plots difficult. to inspect. 


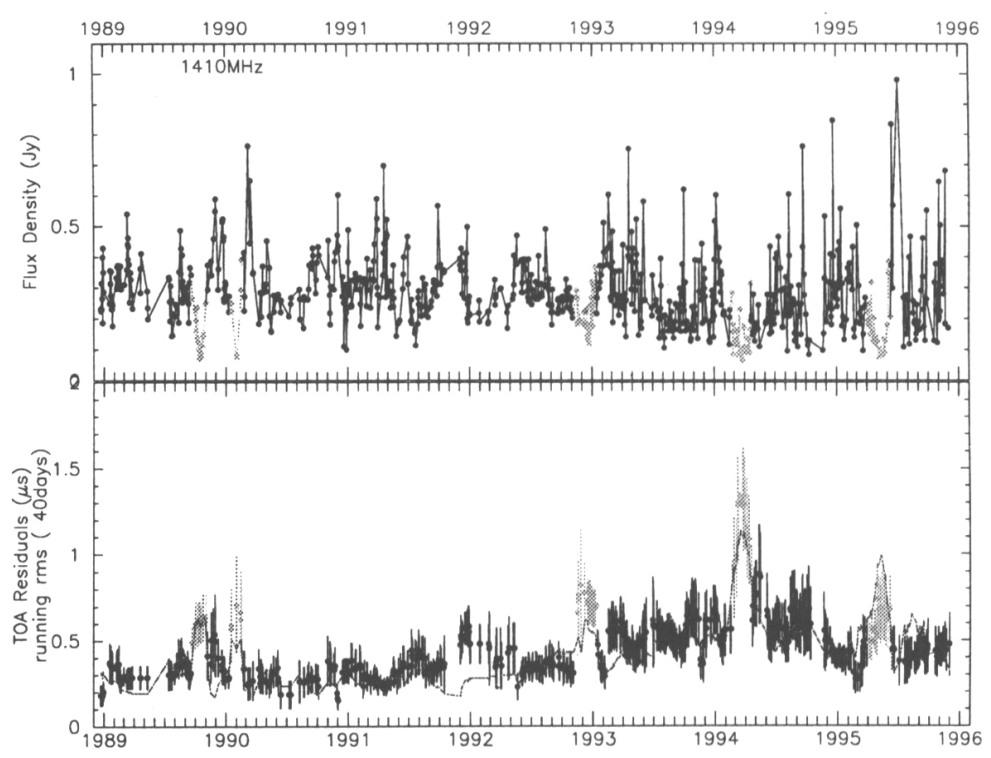

Figure 1. Running rms of the post-fit TOA is plotted along with the flux density variations for the pulsar PSR B1937+21. An estimation of the mean TOA uncertainty depending on the flux density is also plotted. The grayed periods correspond to proposed identification of refractive scintillation events.

For PSR B1937+21, five refractive scintillation events are identified over the 7 year timing series of Nançay. This identification is based on a systematicaly higher TOA post-fit rms when the flux density of the pulsar is depressed as shown in the plots above. The magnitude of the TOA variations is at the level of $2-4 \mu$ s during these events. The durations are $\sim 15$ days for two events and $\sim 1.5$ months for three events. Assuming that the discrete clouds responsible for such events have a logarithmic size distribution, Foster \& Cordes (1990) had speculated that one event of magnitude $\sim 2 \mu$ s are likely to occur once every year.

For PSR B1821-24, the indentification is not so clear. There could be 3 refractive scintillation events in late 1991, February-June 1994 and December 1994. The lower SNR of the observations of PSR B1821-24 and/or the fact that we probe another line of sight could be an explanation to our difficulty to clearly identify refractive scintillation events for this pulsar.

\section{References}

Cognard, I., Bourgois, G., Lestrade, J.-F., Biraud, F., Aubry, D., Darchy, B., Drouhin, J.-P., 1993, Nature, 366, 320

Fiedler, R.L., Dennison, B., Johnston, K.J., Hewish, A., 1987, Nature, 326, 675

Foster, R.S., Cordes, J.M., 1990, ApJ, 364, 123 